\title{
New results on gluon polarisation from COMPASS
}

\author{
Celso Franco on behalf of the COMPASS collaboration \\ LIP - Lisboa \\ Av. Elias Garcia 14 - 1º, 1000-149 Lisboa - Portugal
}

\begin{abstract}
The gluon polarisation in the nucleon has been determined by tagging photon-gluon fusion events via Open-Charm production. The data were taken by the COMPASS Collaboration at CERN between 2002 and 2006, colliding a polarised muon beam on a polarised deuteron target. A new method based on Neural Networks was used to efficiently separate the signal from the uncorrelated background, allowing a new contribution of two low purity channels. At leading order QCD, an average gluon polarisation of $\langle\Delta g / g\rangle_{x}=-0.39 \pm 0.24($ stat $) \pm 0.11($ syst $)$ at a scale $\mu^{2} \approx 13(\mathrm{GeV} / \mathrm{c})^{2}$, and at an average gluon momentum fraction $\langle x\rangle \approx 0.11$, was estimated.
\end{abstract}

\section{Introduction}

Pioneering experiments on the nucleon spin structure obtained an unexpected small quark contribution to the proton spin [2]: $\Delta \Sigma \approx 0.12 \pm 0.17$. This result gave rise to the so called spin crisis, because of the large disagreement as compared to the prediction from the quark model with relativistic corrections $(\Delta \Sigma \approx 0.6)$. This fact triggered extensive studies on the nucleon spin structure, whose results confirmed a small quark contribution with a very good precision [3]:

$$
\Delta \Sigma=0.30 \pm 0.02 \pm 0.01 \quad\left(\text { world data at } Q^{2}=3(\mathrm{GeV} / \mathrm{c})^{2}\right)
$$

Since we know that the nucleon spin must sum to $1 / 2$, the problem was how to solve this spin puzzle. Taking into account the orbital angular momenta, L, of quarks and gluons, the nucleon spin projection (in units of $\hbar$ ) can be decomposed into a sum of its constituents:

$$
S_{z}=\frac{1}{2}=\frac{1}{2} \Delta \Sigma+\Delta G+L_{z}
$$

The first guess to solve this spin puzzle is that a large fraction of the missing nucleon spin comes from the gluons ( $\Delta G$ is the first moment of the gluon helicity distribution). It is a reasonable assumption, since we know from the past that gluons were the solution to the so called momentum crisis in the nucleon (gluons carry $\approx 1 / 2$ of the proton momentum). This was a strong motivation for measuring the gluon contribution to the nucleon spin. Due to a limited range in $Q^{2}$ covered by the polarised experiments, the QCD analysis (through the evolution of DGLAP equations) shows a limited sensitivity to the gluon helicity distribution, $\Delta g(x)$, and to its first moment, $\Delta G$. The determination of $\Delta g(x)$ from QCD evolution must then be complemented by direct measurements in dedicated experiments like COMPASS.

\section{Open-Charm events selection}

In the present analysis $D^{0}$ mesons are used (tagged and untagged with a $D^{*}$ ). In order to be sensitive to the gluon polarisation inside the nucleon, one must tag a process involving a polarised lepton-gluon interaction producing these Open-Charm events (see Figure 1).

DIS 2009 
By neglecting the intrinsic charm content of the nucleon, this Open-Charm method provides, in LO QCD approximation, a clean signature for PGF events (no physical background is present). The main weakness comes from a small charm production crosssection (low statistics).

The COMPASS spectrometer was designed to reconstruct $D^{0}$ mesons, through the invariant mass calculation of their decay products ( $K \pi$ pairs), and for that purpose the $\mathrm{RICH}$ detector plays an important role: requiring proper particle identification, reduces the combinatorial background that masks the real PGF signal centered on the $D^{0}$ mass. Some kinematic cuts are also applied, in the fraction of the virtual photon

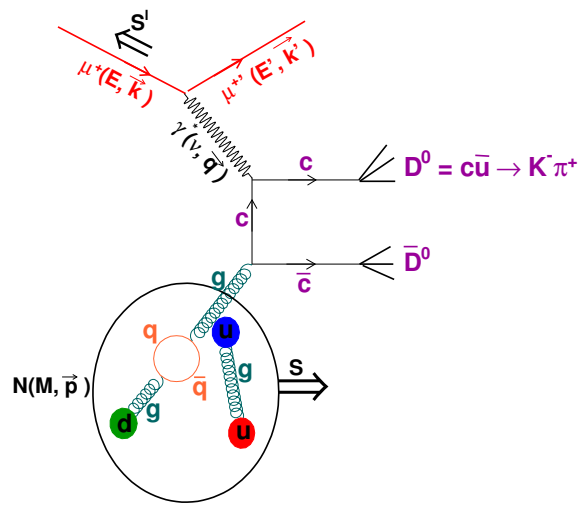
energy carried by the $D^{0}, z_{D^{0}}>0.2$, and in the angle of Kaon decay in the $D^{0}$ center-of-mass, $\left|\cos \left(\theta^{*}\right)\right|<0.65$. Uncorrelated events coming from the fragmentation of the struck quark, from LO processes with light (not charmed) quarks, are collinear with the virtual photon direction, and with a $z_{D^{0}}$ close to zero. The combinatorial background can be further removed by studying the following channel: $D^{*} \rightarrow D^{0} \rightarrow D^{0} \pi_{\text {slow }}$. Applying a cut on the reconstructed $D^{*}$ and $D^{0}$ mass differences, one can check that there is not much room left for the slow pion momenta, resulting in a good signal purity: $3.2 \mathrm{MeV} / \mathrm{c}^{2}<M_{D^{*}}-M_{D^{0}}-M_{\pi}<8.9 \mathrm{MeV} / \mathrm{c}^{2}$. The RICH detector is also used to clean the slow pion sample from electron contamination, allowing for more loose kinematical cuts: $\left|\cos \left(\theta^{*}\right)\right|<0.9$. In this channel one can see a 'bump' in the mass distribution at $\approx-250 \mathrm{MeV} / \mathrm{c}^{2}$, because there is one mode of $D^{0}$ decay involving an extra $\pi^{0}$ which is not detected directly. This 'bump' introduces a new contribution to the analysis, as well as the RICH sub-threshold Kaons samples for the main peak (Kaon candidates not identified by the RICH detector are recovered, as long as they are not positively identified as pions or electrons). This low purity contributions come only from the $D^{*}$ tagged channel (inclusive $D^{0}$ is not clean enough to allow for them). Final samples can be seen on [1], with $8700 D^{0}$ using a $D^{*} \operatorname{tag}, 37400 D^{0}, 6200 D^{0} \rightarrow K \pi \pi^{0}$, and $1800 D^{0}$ with RICH sub-threshold Kaons using $D^{*}$ a tag.

\subsection{Why Open-Charm?}

This channel provides a clean and direct access to the gluon polarisation, as long as the nucleon intrinsic charm contribution is negligible. In COMPASS, the production of $D^{0^{s}}$ are limited to a small $x_{B j}$ range $\left(x_{B j}<0.1\right)$. Theoretical predictions for intrinsic charm shows that COMPASS is not sensitive to this kind of contamination, because they are dominant at higher $x_{B j}$ values (see [4], e.g., for an intrinsic charm prediction assuming nucleon charm fluctuations at a partonic level).

DIS 2009 


\section{Method for gluon polarisation extraction}

To access the gluon polarisation, one can use the asymmetry in the number of Open-Charm events reconstructed inside each target cell (see Figure 3):

$$
A^{e x p}=\frac{n^{u}-n^{d}}{n^{u}+n^{d}}=f P_{\mu} P_{t}\left(\frac{\Delta \sigma}{\sigma}\right)^{\mu N \rightarrow \mu^{\prime} c \bar{c} X}+A_{B}
$$

The number of events inside first cell is defined as $n^{u}$, with $n^{d}$ coming from the second cell polarised in opposite direction.

This experimental asymmetry is proportional to the muon-nucleon asymmetry, taking into consideration some experimental factors (target dilution factor $f$, beam polarisation $P_{\mu}$, and target polarisation $\left.P_{t}\right)$. One can define the differential cross-section for Open-Charm production, in terms of the asymmetries introduced in the previous

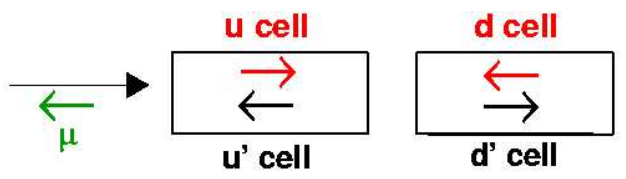

Figure 2: Target scheme (2002-2004 data) equation:

$$
\frac{d n}{d m d X}=a \phi \eta(s+b)\left[1+P_{t} P_{\mu} f\left(\frac{s}{s+b} A_{S}+\frac{b}{s+b} A_{B}\right)\right]
$$

With $m=M_{K \pi}, X$ represents a set of kinematic variables $\left(Q^{2}, Z_{D^{o}}, \ldots\right)$ defining the event, $a$ is the spectrometer acceptance, $\phi$ is the integrated muon beam flux, and $\eta$ the number of target nucleons. $s /(s+b)$ is the signal strength with signal events, $\mathrm{s}(\mathrm{m}, \mathrm{X})$, coming from the invariant mass spectra peak. $\mathrm{s}(\mathrm{m}, \mathrm{X})$ and $\mathrm{b}(\mathrm{m}, \mathrm{X})$ represent the differential unpolarised cross section of signal and background events folded with the experimental resolution.

In LO QCD, $A_{S}$ can be defined as a convolution between the partonic polarised/unpolarised cross section $\left(\Delta \hat{\sigma} / \hat{\sigma}\right.$ for the process $\left.\mu g \rightarrow \mu^{\prime} c \bar{c}\right)$, with the polarised/unpolarised gluon structure function $(\Delta g / g)$ :

$$
\begin{gathered}
A_{S}(X)=\frac{\int \Delta \hat{\sigma} \Delta g F d Y}{\int \hat{\sigma} g F d Y}=a_{L L}(X) \frac{\Delta g}{g}(X) \\
a_{L L}(X)=\frac{\int \hat{a}_{L L} \hat{\sigma} g F d Y}{\int \hat{\sigma} g F d Y}, \quad \frac{\Delta g}{g}(X)=\frac{\int a_{L L} \hat{\sigma} g F \frac{\Delta g}{g} d Y}{\int a_{L L} \hat{\sigma} g F d Y}
\end{gathered}
$$

F describes the fragmentation of c quarks into $D^{0} / D^{*}$ mesons, and the integration is done over the partonic variables, Y, not accessible from the event kinematics, like for example the gluon momentum fraction $x_{g}$. Since the factors $s /(s+b)$ and $a_{L L}$ have a large dispersion, a weighting method was used to minimize the statistical error [3]. All events are weighted with the following signal and background weights: $\omega_{S}=P_{\mu} f a_{L L} s /(s+b)$ and $\omega_{B}=P_{\mu} f D b /(s+$ $b)$, where $\mathrm{D}$ represents the polarisation transfer from the muon to the virtual photon.

In order to minimize acceptance effects between the cells, the target spins were reversed every eight hours: as a result we end up with 2 cells and 4 possible configurations, $t=u, d$, u', d' (Figure 2). One can define 4 equations from Equation 4, for the number of events, and by weighting them with $\omega_{S}$ and then with $\omega_{B}$ we end up with 8 equations and 10 unknowns $\left(\langle\Delta g / g\rangle_{x}, A_{B}\right.$, and the 8 acceptance factors $\alpha_{C}^{t}=\int a^{t} \phi^{t} \eta^{t}(s+b) w_{C} d X$ with $\left.C=S, B\right)$ :

DIS 2009 


$$
\begin{gathered}
\sum_{i=1}^{n_{t}} w_{C, i}=\alpha_{C}^{t}\left(1+\beta_{C}^{t}\left\langle\frac{\Delta g}{g}\right\rangle_{x}+\gamma_{C}^{t} A_{B}\right) \\
\beta_{C}^{t}=\frac{\sum_{i=1}^{n_{t}} P_{t, i} w_{S, i} w_{C, i}}{\sum_{i=1}^{n_{t}} w_{C, i}}, \quad \gamma_{C}^{t}=\frac{\sum_{i=1}^{n_{t}} P_{t, i} w_{B, i} w_{C, i}}{\sum_{i=1}^{n_{t}} w_{C, i}}
\end{gathered}
$$

Since acceptance variations with time affect both cells in same way, the number of unknowns is reduced to $8\left(\alpha_{C}^{u} / \alpha_{C}^{d}=\alpha_{C}^{u^{\prime}} / \alpha_{C}^{d^{\prime}}\right)$, and the system can be solved simultaneously for $\langle\Delta g / g\rangle_{x}$ and $A_{B}$. To solve the system however, one needs $a_{L L}$ and $s /(s+b)$ on a event by event basis.

\subsection{Partonic asymmetry}

This variable $\left(a_{L L}\right)$ is dependent on the full knowledge of the partonic kinematics, which is not directly acessible in the experiment: only one meson is reconstructed for each event. This partonic asymmetry is obtained with the help of a Monte-Carlo generator for heavy flavours (AROMA), in LO, and then it is parameterised with a Neural Network [6] for a final reconstruction on real data. The parameterisation is done through a multidimentional comparison between generated and reconstructed kinematic variables (event by event). The correlation achieved is of the order of $82 \%$ for the main channels, and $76 \%$ for the 3 body decay channel [1].

\subsection{Signal strength}

To obtain the signal strength for each event (signal probabilities), the invariant mass spectrum is divided in several bins of several variables (to account for other dependences than the mass), and then it is fitted inside each bin to obtain $s / b$. Then, a function that is capable of reproducing all signal purities obtained from the fit, inside all bins, is used to get $s /(s+b)$ for every event. This function results from an iterative procedure over all bins inside each variable, where the obtained signal purities are adjusted to the fit values until convergence [5]. Concerning the new low purity channels, a new method had to be introduced, because the signal is too weak to allow for fits in several bins of several variables. A Neural Network is used as a qualification of events [6]: they are sorted according to similar kinematic dependences, and also signal identification is possible on a event by event basis. Two real data sets are compared: a good charge combination sample, gcc (real $D^{0}$ spectrum), is compared with a wrong charge combination one, wcc (background model). The gcc sample is tagged as a signal model, $t=0.95$, and the wcc sample is tagged as $t=0.05$ (Network background interpretation). For wcc, a wrong charge on the $D^{0}$ decay products is applied to avoid the presence of this mesons. The multidimentional Neural Network comparison is performed using the shapes of several kinematic variables distributions [1] (gcc vs. wcc): during the training procedure an answer $o_{1}$ is calculated for each event (trying to minimize the error $\left.\left(t-o_{1}\right)^{2}\right)$, and if the background model is good enough the Network is able to identify the signal events. To ensure universality a testing set is also used, and if the errors start to diverge (compared to the training set) the learning strategy is changed: redundant neurons are killed and new ones are allowed to be born. Eventually the training stops when the error stabilizes, by frozing all the weights of the variable-neuron connections (Network structure).

DIS 2009 
Finally, $s /(s+b)$ is obtained as before, but in this case only the final Network response, $o_{1}$, is used as a variable (reflecting events with the same kinematic dependences). The probability behaviour achieved in the invariant mass spectrum can be checked on [1] (bins of parameterised $s /(s+b)$ ), allowing for softer kinematical cuts: this implies more signal and background selected, but with an efficient $s /(s+b)$ parameterisation, all signal is shifted to higher probability bins (better precision on the gluon polarisation measurement).

\section{Results}

Since $\Delta g / g(x)$ is approximately linear dependent on $x$ in the range covered, we end up with a measurement of $\Delta g / g(\langle x\rangle)$ (where $\langle x\rangle$ is calculated using the signal weights). The final value, using the method described above is ( $A_{B}$ was found to be consistent with zero):

$$
\left\langle\frac{\Delta g}{g}\right\rangle_{x}=-0.39 \pm 0.24(\text { stat }) \pm 0.11(\text { syst }) @ \mu^{2} \approx 13(\mathrm{GeV} / \mathrm{c})^{2},\left\langle x_{g}\right\rangle \approx 0.11
$$

\section{Conclusion}

A direct measurement of $\langle\Delta g / g\rangle_{x}$ was extracted from the asymmetry on $D^{0}$ meson production, with a statistical precision of 0.24 . The systematic contribution is smaller and estimated to be 0.11 . The result was obtained in LO QCD, for the PGF process, and it can be compared with the high-pt results (from COMPASS and other experiments) in Figure 3. The Open-Charm point is consistent with the other measurements, favouring small values of $\langle\Delta g / g\rangle_{x}$. The scale of this analysis is $\mu^{2} \approx 13(\mathrm{GeV} / \mathrm{c})^{2}$, while all the other points and curves are at a scale $\mu^{2} \approx 3(\mathrm{GeV} / \mathrm{c})^{2}$. The result shown is weakly model dependent (Monte-Carlo).

\section{References}

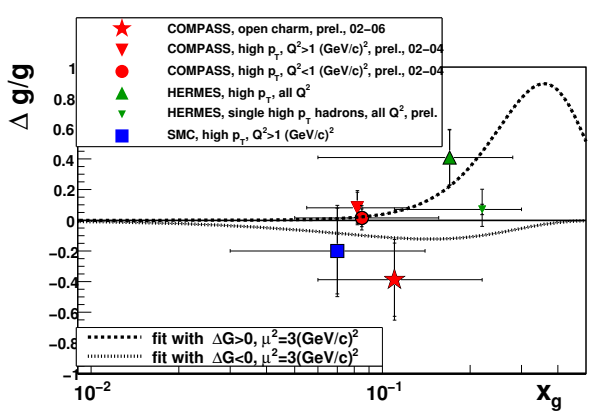

Figure 3: Comparison of $\Delta g / g$ measurements obtained from Open-Charm and high-pt analysis. The curves display two parameterisations from COMPASS QCD analysis at NLO [3].

[1] Slides: http://indico. cern. ch/getFile.py/access? contribId=306\&sessionId=4\&resId=0\&material Id=slides\&conf Id=53294

[2] EMC, J. Ashman et al., Nucl. Phys. B 206 (1988) 364

[3] COMPASS, V. Yu. Alexakhin et al., Phys. Lett. B 647 (2007) 8

[4] S.J. Brodsky, et al., Phys. Lett. B 93 (1980) 451

[5] COMPASS, V. Alekseev, et al., Phys. Lett. B 676 (2009) 31

[6] R. Sulej et al. Measur.Sci.Tech.18: 2486-2490, 2007

DIS 2009 